\title{
CT and PET Registration Using Deformations Incorporating Tumor-Based Constraints
}

\author{
Antonio Moreno ${ }^{1,2}$, Gaspar Delso $^{3}$, Oscar Camara ${ }^{4}$, and Isabelle Bloch ${ }^{1}$ \\ 1 Ecole Nationale Supérieure des Télécommunications, TSI Department, \\ CNRS UMR 5141, 46 rue Barrault, 75634, Paris Cedex 13, France \\ Antonio.Moreno@enst.fr \\ 2 Segami, 22 avenue de la Sibelle, F-75014 Paris, France \\ 3 Philips Medical Systems, Suresnes, France \\ 4 Center for Medical Image Computing, Department of Medical Physics, \\ University College London, London WC1E 6BT, UK
}

\begin{abstract}
Registration of $\mathrm{CT}$ and PET thoracic images has to cope with deformations of the lungs during breathing. Possible tumors in the lungs usually do not follow the same deformations, and this should be taken into account in the registration procedure. We show in this paper how to introduce tumor-based constraints into a non-linear registration of thoracic CT and PET images. Tumors are segmented by means of a semi-automatic procedure and they are used to guarantee relevant deformations near the pathology. Results on synthetic and real data demonstrate a significant improvement of the combination of anatomical and functional images for diagnosis and for oncology applications.
\end{abstract}

\section{Introduction}

Computed Tomography (CT) and Positron Emission Tomography (PET), particularly dealing with thoracic and abdominal regions, furnish complementary information about the anatomy and the metabolism of human body. Their combination has a significant impact on improving medical decisions for diagnosis and therapy [3] even with the combined PET/CT devices where registration remains necessary to compensate patient respiration and heart beating. In particular, accuracy is fundamental when there is pathology.

Registration of these two modalities is a challenging application due to the poor quality of the PET image and the large deformations involved in these regions.

Most of the existing methods have as a limitation that regions placed inside or near the main structures will be deformed more or less according to the registration computed for the latter, depending on how local is the deformation. A critical example of this situation occurs when a tumor is located inside the lungs and there is a large volume difference between CT and PET images (due to the breathing). In this case, the tumor can be registered according to the transformation computed for the lungs, taking absurd shapes, such as shown in Figure 1. Therefore, the aim of this paper is to avoid this undesired tumor 
misregistrations in order to preserve tumor geometry and, in particular, intensity since it is critical for clinical studies, for instance based on SUV (Standardized Uptake Value).

In Section 2, we summarize existing work related to this subject and we provide an overview of the proposed approach. In Section [3, we describe the segmentation of the targeted structures, i.e., the body, the lungs and the tumors. The introduction of tumor-based constraints into the registration algorithm is detailed in Section 4. Section 5 presents some results obtained on synthetic and real data. Finally, conclusions and future works are discussed in Section 6 .
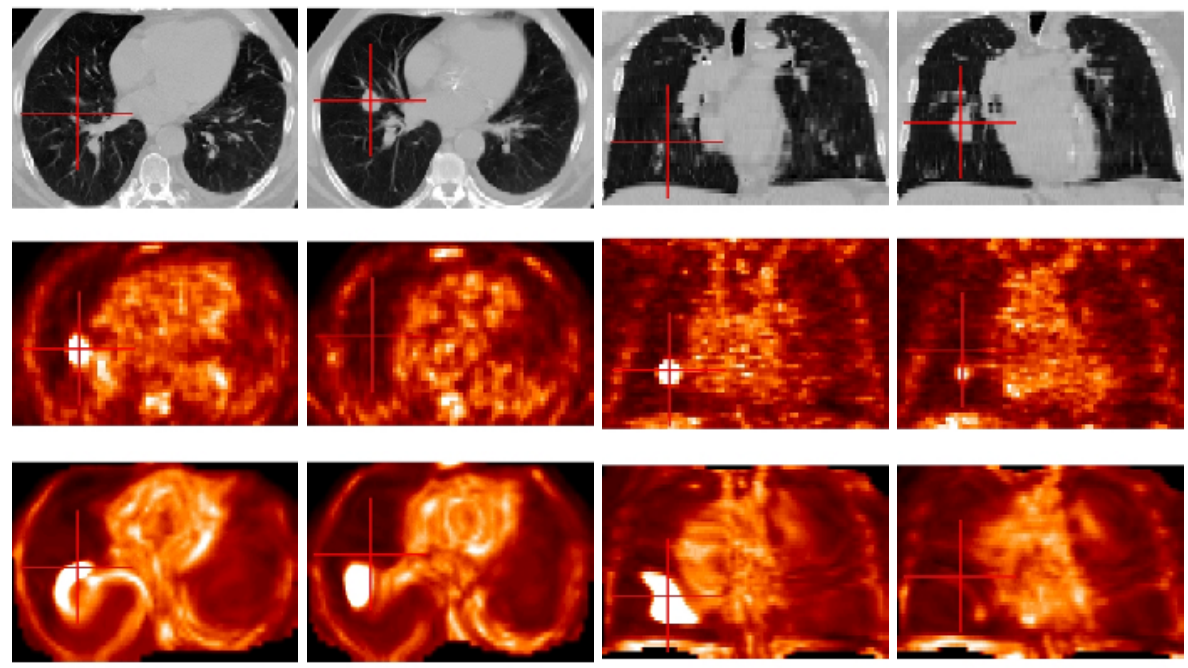

Fig. 1. Axial and coronal slices in CT (first row) and in PET (second row). Result of the non-linear registration without tumor-based constraints (third row). The absence of these constraints leads to undesired and irrelevant deformations of the pathology. On the images of the first and third columns, the cursor is positioned on the tumor localization in PET data, while in the second and fourth columns, it is positioned on the tumor localization in CT data. This example shows an erroneous positioning of the tumor and illustrates the importance of tumor segmentation and the use of tumor-specific constraints.

\section{Related Work and Overview of the Proposed Approach}

Some approaches have already been developed for registration of multimodality images in pathological cases (pulmonary nodules, cancer), such as in [5]. However these approaches compute a rigid (or affine) registration for all the structures and they do not take into account the local nature of the deformations.

Rohlfing and Maurer [9] have developed a method of non-rigid registration based on B-spline Free-Form Deformations as in [1], but they have added some incompressibility constraints (using the properties of the Jacobian) which only 
guarantee the preservation of the volume of the structures but not their shape. Loeckx et al. [10, have added a local rigidity constraint and they have obtained very promising results.

A different approach, that we consider closer to physical reality of human body, is based on the combination of rigid and non-rigid deformations, as suggested by Little et al. [7] and Huesman et al. 6]. These methods are based on the use of point interpolation techniques, together with a weighting of the deformation according to a distance function. Castellanos et al. 8] developed a slightly different methodology, in which local non-rigid warpings are used to guarantee the continuity of the transformation.

The advantage of the approach by Little is that it takes into account rigid structures and the deformations applied to the image are continuous and smooth. The method we propose is inspired by this one and adapted to develop a registration algorithm for the thoracic region in the presence of pathologies.

The data consist of 3D CT and PET images of pathological cases, exhibiting tumors in the lungs. We assume that the tumor is rigid and thus a linear transformation is sufficient to cope with its movements between CT and PET images. This hypothesis is relevant and in accordance with the clinicians' point of view, since tumors are often a compact mass of pathological tissue. In order to guarantee a good registration of both normal and pathological structures, the first step consists of a segmentation of all structures which are visible in both modalities. Then we define two groups of landmarks in both images, which correspond to homologous points, and will guide the deformation of the PET image towards the CT image. The positions of the landmarks are therefore adapted to anatomical shapes. This is an important feature and one of the originalities of our method. The deformation at each point is computed using an interpolation procedure based on the landmarks, on the specific type of deformation of each landmark depending on the structure it belongs to, and weighted by a distance function, which guarantees that the transformation will be continuous.

The proposed approach has two main advantages:

1. As the transformation near the tumor is reduced by using the distance weight, even if we have some small errors in the tumor segmentation (often quite challenging, mainly in $\mathrm{CT}$ ), we will obtain a consistent and robust transformation.

2. In the considered application, one important fact is that the objects to register are not the same in the two images. For instance, the volume of the "anatomical" tumor in CT is not necessarily the same as the volume of the "functional" tumor in PET because the two modalities highlight different characteristics of the objects. The registration of these two views of the tumor must preserve these local differences, which can be very useful because we could discover a part of the anatomy that is touched by the pathology and could not be seen in the CT image. This also advocates in favor of a rigid local registration. 


\section{Segmentation}

The first stage of our method consists in segmenting the most relevant structures that can be observed in both modalities. In this paper, we have segmented the body contours and the lungs. The body is segmented using automatic thresholding and mathematical morphology operators. Lung segmentation is achieved using the procedure introduced in 2 based on a hierarchical method that uses mathematical morphology guided by the previously segmented structures. These structures will be the base for our algorithm as landmarks will lean on them.

Nevertheless, the most important objects to segment are the tumors. In a first approach, tumors have been segmented by a semi-interactive segmentation algorithm, using the coordinates furnished by a "click" of an expert inside the pathology. More precisely, the interaction consists for the physician in defining a seed-point in the tumor of interest (in both CT and PET images). Next, both selected points are used as the input to a relaxation region growing algorithm 4. This semi-interactive approach has been chosen due to the complexity of a fully automatic tumor segmentation method, mainly in CT images. In addition, this very reduced interaction is well accepted by the users, and even required because it is faster than any non-supervised method and it assures consistent results.

The segmented tumors in CT and PET images are used in the following in order to:

1. calculate the rigid transformation (translation) of the tumor from PET image (source image) to CT image (target image);

2. calculate the distance map to the tumor in PET that will constrain the deformation to be rigid inside the tumor and increasingly non-rigid away from it.

Figure 2 shows some results of the body contour, lungs and tumor segmentations.
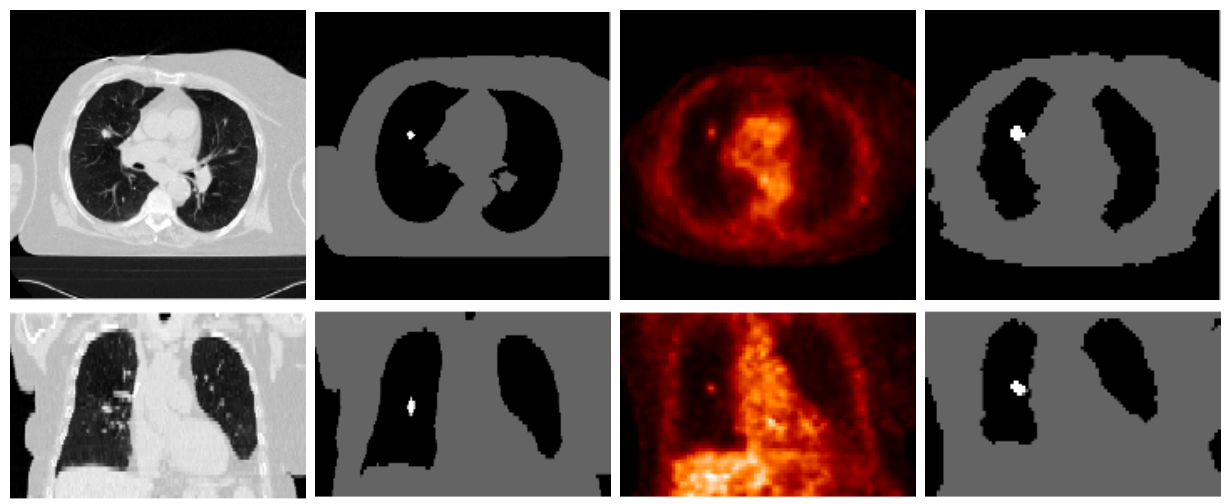

Fig. 2. Segmentation results. First and third columns: original CT and PET images (axial and coronal views). Second and fourth columns: results of the segmentation of the body contour, the lungs and the tumor in both modalities. 


\section{Combining Rigid and Non-linear Deformations Using a Continuous Distance Function}

Based on pairs of corresponding landmarks in both images, the transformation is interpolated through the whole image using the approach in [7]. We introduce the rigid structure constraint so that the non-rigid transformation is gradually weighted down in the proximity of predefined rigid objects.

In this paper, we apply this theoretical framework to a particular 3D case dealing with just one rigid structure (only one tumor is present in each image).

\subsection{Point-Based Displacement Interpolation}

The first step in a point-based interpolation algorithm concerns the selection of the landmarks guiding the transformation. Thus, homologous structures in both images are registered based on landmarks points defined on their surface. The resulting deformation will be exact at these landmarks and smooth elsewhere, which is achieved by interpolation.

Let us denote by $\boldsymbol{t}_{i}$ the $n$ landmarks on the source image that we want to transform to new sites $\boldsymbol{u}_{i}$ (the homologous landmarks) in the target image.

The deformation at each point $\boldsymbol{t}$ in the image is defined as:

$$
\boldsymbol{f}(\boldsymbol{t})=\mathcal{L}(\boldsymbol{t})+\sum_{j=1}^{n} B_{j}^{T} \sigma\left(\boldsymbol{t}, \boldsymbol{t}_{j}\right)
$$

under the constraints

$$
\forall i, \quad \boldsymbol{u}_{i}=\boldsymbol{f}\left(\boldsymbol{t}_{i}\right)
$$

The first term, $\mathcal{L}(\boldsymbol{t})$, represents the linear transformation of every point $\boldsymbol{t}$ in the source image. The second term represents the non-linear transformation which is, for a point $\boldsymbol{t}$, the sum of $n$ terms, one for each landmark. Each term is the product of the coefficients of a matrix $B$ (that will be computed in order to satisfy the constraints on the landmarks) with a function $\sigma\left(\boldsymbol{t}, \boldsymbol{t}_{j}\right)$, depending on the distance between $\boldsymbol{t}$ and $\boldsymbol{t}_{j}$ :

$$
\sigma\left(t, t_{j}\right)=\left|t-t_{j}\right|
$$

This form has favorable properties for image registration [1]. However, different functions can be used as the one described in [7].

With the constraints given by Equation 2, we can calculate the coefficients $B$ of the non-linear term by expressing Equation 1 for $\boldsymbol{t}=\boldsymbol{t}_{i}$. The transformation can then be defined in a matricial way:

$$
\Sigma B+L=U
$$

where $U$ is the matrix of the landmarks $\boldsymbol{u}_{i}$ in the target image (the constraints), $\Sigma_{i j}=\sigma\left(\boldsymbol{t}_{i}, \boldsymbol{t}_{j}\right)$ (given by Equation 3), $B$ is the matrix of the coefficients of the non-linear term and $L$ represents the application of the linear transformation to 
the landmarks in the source image, $\boldsymbol{t}_{i}$. In our specific case, this linear transformation $L$ is the translation of the tumor (between PET and CT images) found in the preprocessing.

From Equation 4, the matrix $B$ is obtained as:

$$
B=\Sigma^{-1}(U-L) .
$$

Once the coefficients of $B$ are found, we can calculate the general interpolation solution for every point in $\mathbb{R}^{3}$ as shown in Equation 1

\subsection{Introducing Rigid Structures}

In this section, we show how to introduce the constraint imposed by the rigid structures in the images. As mentioned in Section 2, the tumor has not exactly the same size nor the same shape in PET and CT images. However, we know that they correspond to the same structure and we register them in a linear way (translation defined by the difference of their centers of mass).

To add the influence of the rigid structure $O$, we have redefined the function $\sigma\left(\boldsymbol{t}, \boldsymbol{t}_{j}\right)$ as $\sigma^{\prime}\left(\boldsymbol{t}, \boldsymbol{t}_{j}\right)$ in the following way:

$$
\sigma^{\prime}\left(\boldsymbol{t}, \boldsymbol{t}_{j}\right)=d(\boldsymbol{t}, O) d\left(\boldsymbol{t}_{i}, O\right) \sigma\left(\boldsymbol{t}, \boldsymbol{t}_{j}\right)
$$

where $d(\boldsymbol{t}, O)$ is a distance function from point $\boldsymbol{t}$ to object $O$. It is equal to zero for $\boldsymbol{t} \in O$ (inside the rigid structure) and takes small values when $\boldsymbol{t}$ is near the structure. This distance function is continuous over $\mathbb{R}^{3}$ and it weights the function $\sigma\left(\boldsymbol{t}, \boldsymbol{t}_{j}\right)$ (see Equation 3). So the importance of the non-linear deformation will be controlled by the distance to the rigid object in the following manner:

- $d(\boldsymbol{t}, O)$ makes $\sigma^{\prime}\left(\boldsymbol{t}, \boldsymbol{t}_{j}\right)$ tend towards zero when the point for which we are calculating the transformation is close to the rigid object;

- $d\left(\boldsymbol{t}_{i}, O\right)$ makes $\sigma^{\prime}\left(\boldsymbol{t}, \boldsymbol{t}_{j}\right)$ tend towards zero when the landmark $\boldsymbol{t}_{j}$ is near the rigid object. This means that the landmarks close to the rigid structure will hardly contribute to the non-linear transformation computation.

Equation 4 is then rewritten by replacing $\Sigma$ by $\Sigma^{\prime}$, leading to a new matrix $B^{\prime}$. Finally, we can calculate the general interpolation solution for every point in $\mathbb{R}^{3}$ as in Equation 1

\section{Results}

We present in this section some results that we have obtained on synthetic images, on segmented images and, finally, on real images.

\subsection{Synthetic Images}

This first experiment on synthetic images aims at checking that the rigid structures are transformed rigidly, that the landmarks are correctly translated too and, finally, that the transformation elsewhere is consistent and smooth. 
This simulation was designed to be similar to the effect we can find with real images. The rigid structure is the "tumor" and it is just translated. The frame of our synthetic images simulate the contour of the body and the internal black square replace the lungs. As we are taking the PET image as the one to be deformed (source image), we simulate an expansive transformation because the lungs in PET are usually smaller than in CT images. This is due to the fact that the CT image is often acquired in maximal inspiration of the patient. The result in this case is shown in the second row of Figure 3 .
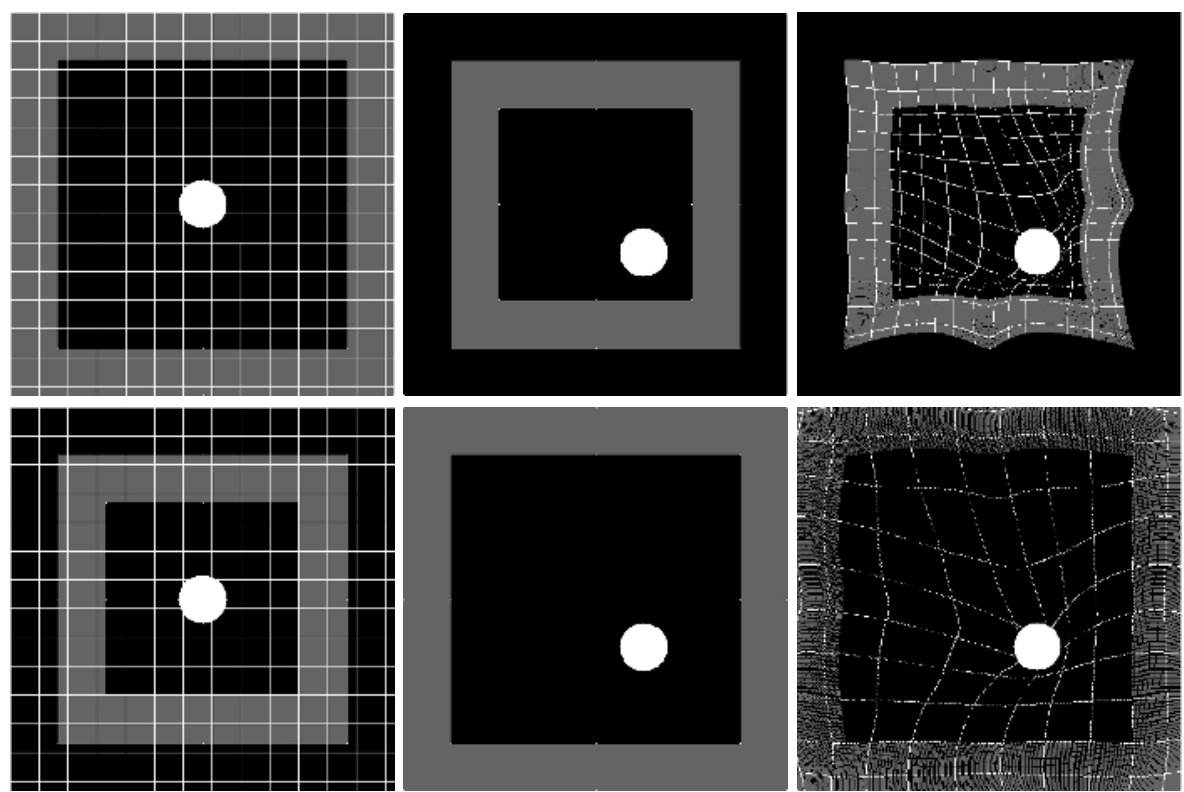

Fig. 3. Results on synthetic images. First row: effects of shrinking a frame (in grey in the figure) and translating the "tumor" (in white in the figure). Second row: effects of expanding a frame and translating the "tumor". Source images (with a grid) are shown on the left, target images are in the middle and the results of the transformation on the right. The landmarks are located on the internal and external edges of the frame in grey (on the corners and in the middle of the sides). The total number of landmarks is 16 in both examples.

In order to observe the transformation all over the image, we have plotted a grid on it. To illustrate the effect of the transformation we have simulated a compression and an expansion of a frame and a simple translation of the "tumor". It can be seen in Figure 3 that the results with our synthetic images are satisfactory as the shape of the rigid structure (the "tumor") is conserved and the landmarks are translated correctly. The frame, on which the landmarks are put, is deformed in a continuous and smooth way. If we do not apply the constraints on the rigid structure we obtain an undesired transformation as illustrated in Figure 4 (the tumor is expanded). 

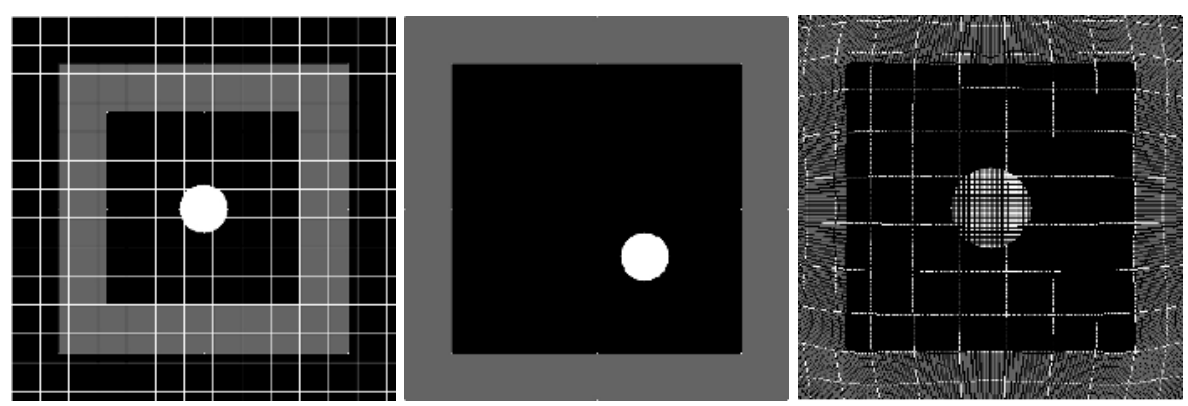

Fig. 4. Result on a synthetic image without constraints on the rigid structure when we apply an expansion to the frame using 16 landmarks. Source image (with a grid) is shown on the left, target image is in the middle and the result of the transformation on the right.

However, it must be noticed that the edges of the frame are not totally straight after the transformation. In general, the more landmarks we have, the better the result will be. The positions of the landmarks are important too. Here we have chosen to spread them uniformly over the internal and external edges of the frame.

The algorithm has also been tested on 3D synthetic images with similar results. We only show here the results on bi-dimensional images for the sake of simplicity.

\subsection{Segmented Images}

In order to appreciate more clearly the effect of the transformation, we have first used the results of the segmentation to create the simplified real images. They are not only useful to analyze the deformation but it is also easier to define the landmarks on them.

Landmarks have to correspond to the same anatomical reality in both images. Thus we have decided to place them (uniformly distributed) on the surfaces of the lungs.

Figure 5 shows some results on the simplified images. A grid is superimposed on the segmented PET image for better visualization. In these cases, we have fixed the corners of the images to avoid undesired deformations. In Figure 6. we can see the undesired effect produced if there is no landmark to retain the borders of the image.

For any number of landmarks, the tumor is registered correctly with a rigid transformation. Nevertheless, the quality of the result depends on the quantity of landmarks and their positions. If the number of landmarks is too low, the algorithm does not have enough constraints to find the desired transformation.

Here the results are obtained by applying the direct transformation in order to better appreciate the influence of the deformation in every region of the image. However it is clear that the final result should be based on the computation of 


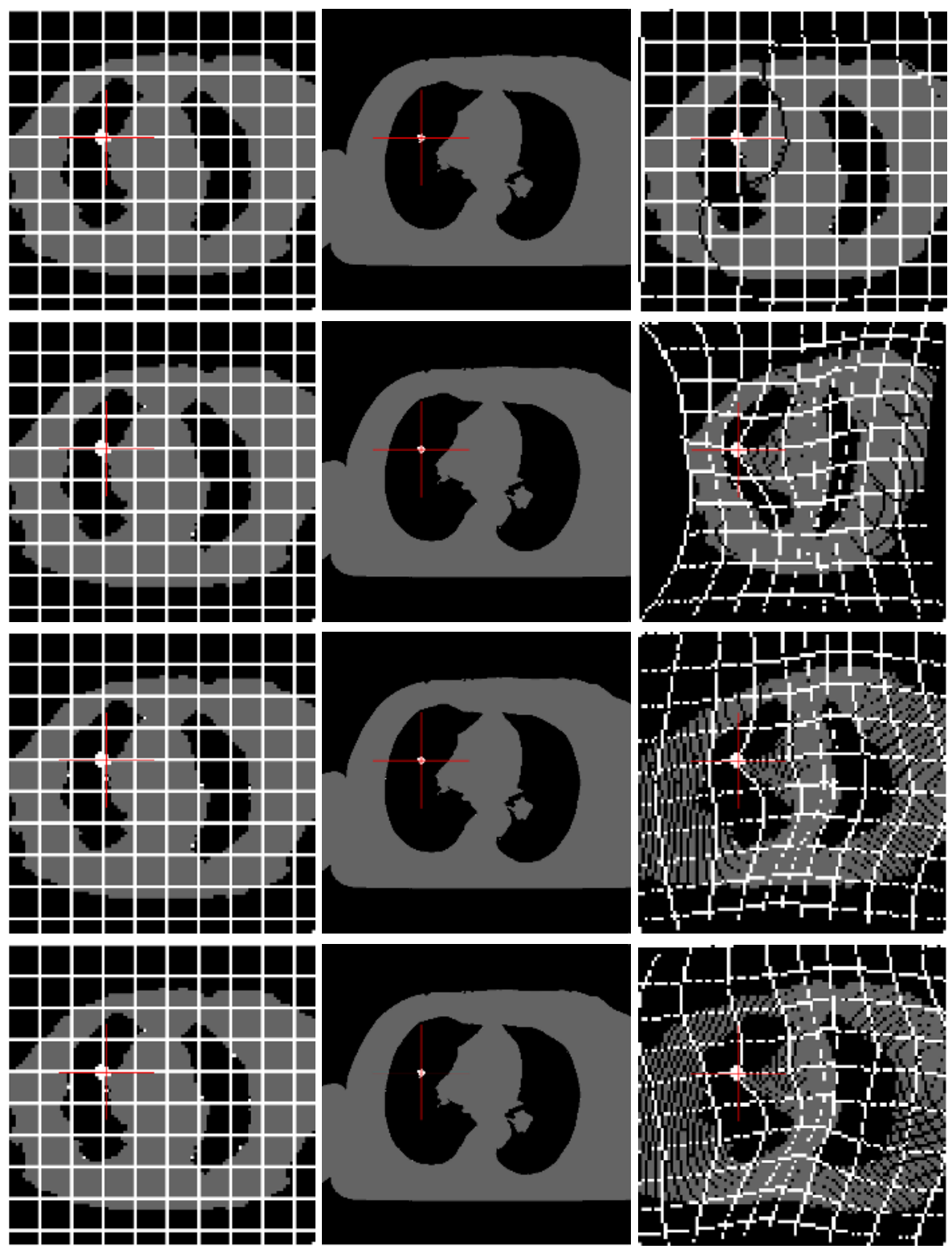

Fig. 5. Results on simplified images. First column: segmented PET images with a grid for visualization purpose (landmarks are also marked in white). Second column: segmented CT images with the corresponding landmarks. Third column: results of the registration of the simplified PET and $\mathrm{CT}$ images. In the first row 4 landmarks have been used (fixed on the corners of the image). Then additional landmarks are chosen on the walls of the lungs (uniformly distributed): 4 in the second line, 8 in the third one and 12 in the last one. In all the images the cursor is centered on the tumor in the CT image. 

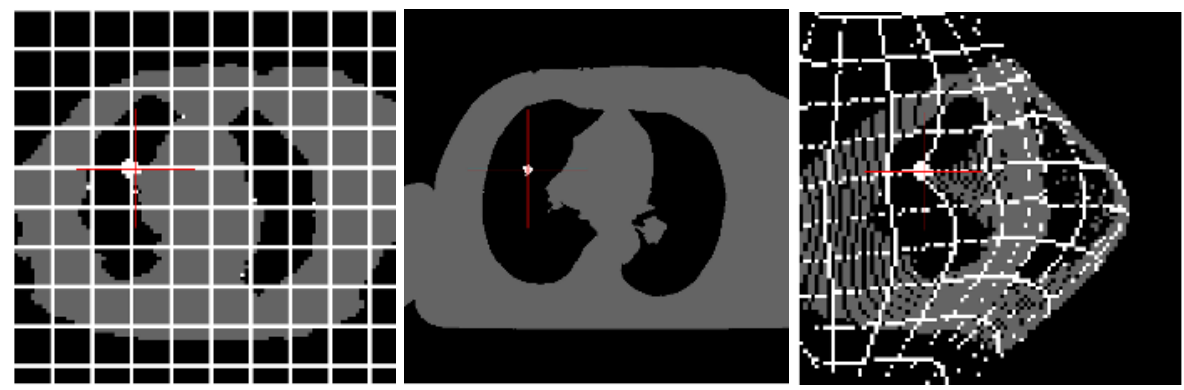

Fig. 6. Result on the simplified images. This is the kind of result we obtain if we do not fix the corners of the image. Here we have only 8 landmarks on the walls of the lungs.
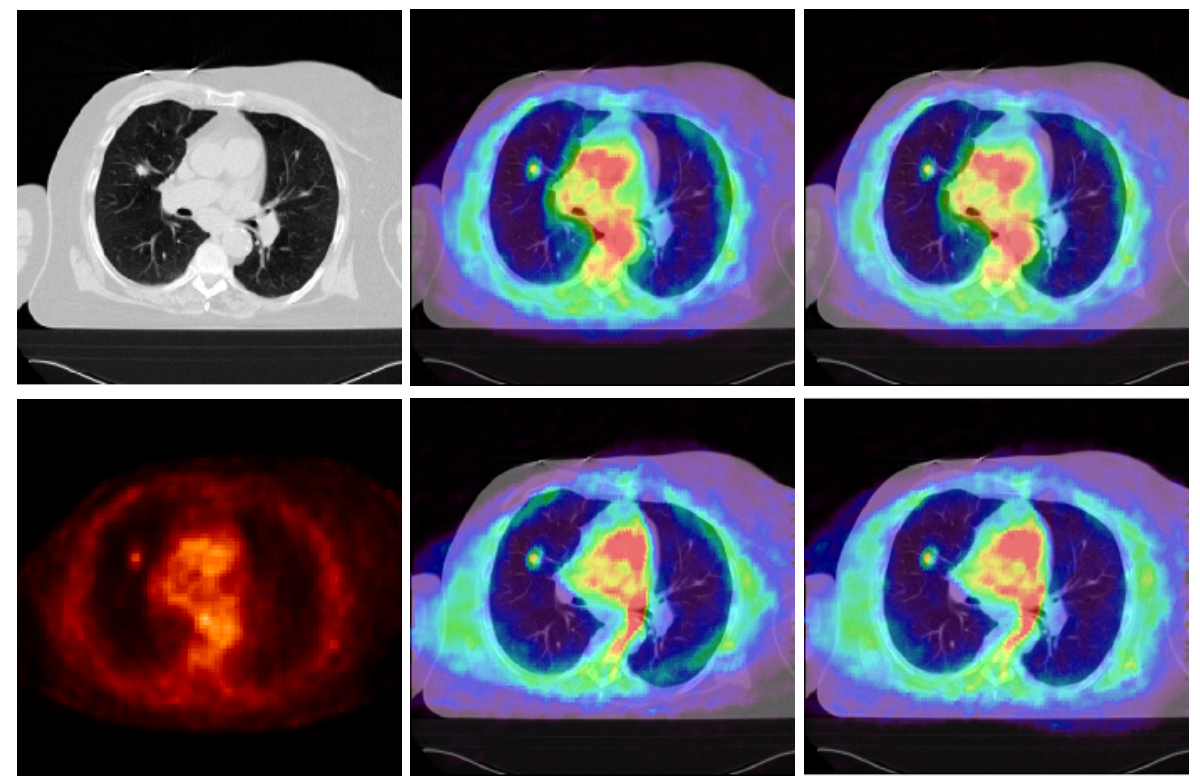

Fig. 7. Results on real images. The CT image and the original PET image are shown in the first column. Second and third columns, from left to right and from top to bottom: superimposition of the CT image with the deformed PET image with 0 (only global translation), 4, 12 and 16 landmarks.

the inverse transformation at each point of the result image in order to avoid unassigned points.

\subsection{Real Images}

Figure 7 shows the results on real images. As happened with the simplified images, we have to fix the corners of the images to avoid misregistrations. 
As previously, the tumor is registered correctly with a rigid transformation in all the cases. However, the accuracy of the registration depends on the number and the distribution of the landmarks. If the number of landmarks is not sufficient there are errors. It can be seen that with an appropriate number of landmarks the registration is very satisfactory. Figure 7 shows that with only 16 landmarks in CT and in PET, the results are good and the walls of the lungs are perfectly superimposed. The results are considerably improved, compared to those obtained with 4 or 12 landmarks.

This shows that the minimal number of landmarks does not need to be very large if the landmarks are correctly distributed, i.e., if they are located on the points that suffer the most important deformations.

\section{Conclusion and Future Work}

We have developed a $\mathrm{CT} / \mathrm{PET}$ registration method adapted to pathological cases. It consists in computing a deformation of the PET image guided by a group of landmarks and with tumor-based constraints. Our algorithm avoids undesired tumor misregistrations and it preserves tumor geometry and intensity.

One of the originalities of our method is that the positions of the landmarks are adapted to anatomical shapes. In addition to this, as the transformation near the tumor is reduced by the distance weight, even if the tumor segmentation is not perfect, the registration remains consistent and robust. Moreover, the tumor in CT and PET has not necessarily the same size and shape, therefore the registration of these two modalities is very useful because all the information of the PET image is preserved. This is very important in order to know the true extension of the pathology for diagnosis and for the treatment of the tumor with radiotherapy, for example.

Future work will focus on the automatic selection of the landmarks in order to furnish a consistent distribution on the surfaces of the structures and to guarantee a satisfactory registration.

A comparison with other methods (as Loeckx's one) will provide some conclusions on the limits of each method and their application fields.

Although validation is a common difficulty in registration [12, we plan an evaluation phase in collaboration with clinicians.

\section{Acknowledgments}

The authors would like to thank Liège, Lille, Louisville and Val de Grâce Hospitals for the images and helpful discussions and the members of Segami Corporation for their contribution to this project. This work was partially supported by the French Ministry for Research. 


\section{References}

1. O. Camara-Rey: "Non-Linear Registration of Thoracic and Abdominal CT and PET Images: Methodology Study and Application in Clinical Routine", PhD dissertation, ENST (ENST 2003 E 043), Paris, France, December 2003

2. G. Delso: "Registro Elástico de Imágenes Médicas Multimodales. Aplicación en Oncología", PhD dissertation, Centre de Recerca en Enginyeria Biomèdica, Universitat Politècnica de Catalunya, Barcelona, Spain, October 2003

3. H.N. Wagner, Jr., MD: "Creating Lifetime Images of Health and Disease", 2004 SNM Highlights Lecture, pp. $11 \mathrm{~N}-41 \mathrm{~N}$ and "PET and PET/CT: Progress, Rewards, and Challenges", The Journal of Nuclear Medicine, Vol. 44, No. 7, pp. $10 \mathrm{~N}-14 \mathrm{~N}$, July 2003

4. R. Adams, and L. Bischof: "Seeded region growing", IEEE Transactions on Pattern Analysis and Machine Intelligence, 16, pp. 641-647, 1994

5. T. Blaffert, and R. Wiemker: "Comparison of different follow-up lung registration methods with and without segmentation", Medical Imaging 2004, Proceedings of SPIE Vol. 5370, pp. 1701-1708

6. R.H. Huesman, G.J. Klein, J.A. Kimdon, C. Kuo, and S. Majumdar: "Deformable Registration of Multimodal Data Including Rigid Structures", IEEE Transactions on Nuclear Science, Vol. 50, No. 3, June 2003

7. J.A. Little, D.L.G. Hill, and D.J. Hawkes: "Deformations Incorporating Rigid Structures", Computer Vision and Image Understanding, Vol. 66, No. 2, pp. 223232, May 1997

8. N.P. Castellanos, P.L.D. Angel, and V. Medina: "Nonrigid medical image registration technique as a composition of local warpings", Pattern Recognition 37, pp. 2141-2154, 2004

9. T. Rohlfing, and C.R. Maurer: "Intensity-Based Non-rigid Registration Using Adaptive Multilevel Free-Form Deformation with an Incompressibility Constraint", Proceedings of MICCAI 2001, LNCS 2208, pp. 111-119, 2001

10. D. Loeckx, F. Maes, D. Vandermeulen, and P. Suetens: "Nonrigid Image Registration Using Free-Form Deformations with Local Rigidity Constraint", Proceedings of MICCAI 2004, LNCS 3216, pp. 639-646, 2004

11. R. Wiemker, K. Rohr, L. Binder, R. Sprengel, and H.S. Stiehl: "Application of elastic registration to imagery from airbone scanners", Congress of the International Society for Photogrammetry and Remote Sensing (ISPRS'96), pp. 949-954, 1996

12. J.A. Schnabel, C. Tanner, A.D. Castellano-Smith, A. Degenhard, M.O. Leach, D.R. Hose, D.L.G. Hill, and D.J. Hawkes: "Validation of Nonrigid Image Registration Using Finite-Element Methods: Application to Breast MR Images", IEEE Transactions on Medical Imaging, Vol. 22, No. 2, February 2003 